\title{
CORRIGENDUM
}

\section{BAX inhibitor-1 enhances cancer metastasis by altering glucose metabolism and activating the sodium-hydrogen exchanger: the alteration of mitochondrial function}

G-H Lee, C Yan, S-J Shin, S-C Hong, T Ahn, A Moon, SJ Park, YC Lee, WH Yoo, H-T Kim, D-S Kim, S-W Chae, H-R Kim and H-J Chae

Oncogene (2016) 35, 803-804; doi:10.1038/onc.2015.421

Correction to: Oncogene (2010) 29, 2130-2141; doi: 10.1038/ onc.2009.491

Since the publication of the above article the authors have identified an error in the labelling of Figure $4 \mathrm{c}$ and in the listing of the siRNA sequence of $\mathrm{BI}-1$ in the Materials and Methods section.

Shown here is the corrected version of Figure $4 \mathrm{c}-\mathrm{BI}-1$, and Neo labelling has been reversed.

The below paragraph from the Materials and Methods section of the paper has been amended to correct the listing of the siRNA sequence for $\mathrm{BI}-1$.

siRNA transfection

The siRNAs were synthesized in duplex and purified forms using Bioneer technology (Daejon, South Korea). The double-strand small interfering RNAs (siRNAs) targeting BI-1 (for Bl-1 siRNA, 5'-GUGCUAUCCUACGAAAUCA-3' (forward) and 5'-UGAUUUC GUAGGAUAGCAC-3' (reverse); for non-specific siRNA, 5'-CUGA ACAACCAAUGCAAAU-3' (forward) and 5'-AUUUGCAUUGGUUGU UCAG-3' (reverse)) were transfected separately or together into Neo and Bl-1 cells using Amaxa Nucleofector (Amaxa, Gaithersburg, MD, USA). Briefly, confluent cells were trypsinized and resuspended in Amaxa Nucleofector solution at $2 \times 10^{5}$ cells per $100 \mu \mathrm{l}$ of solution, and BI-1 or on-specific siRNA was added. Cells were transfected by electroporation using the A24 pulsing program.

The authors wish to apologise for any inconvenience caused and confirm that the conclusions drawn from this research are not affected by this error. 

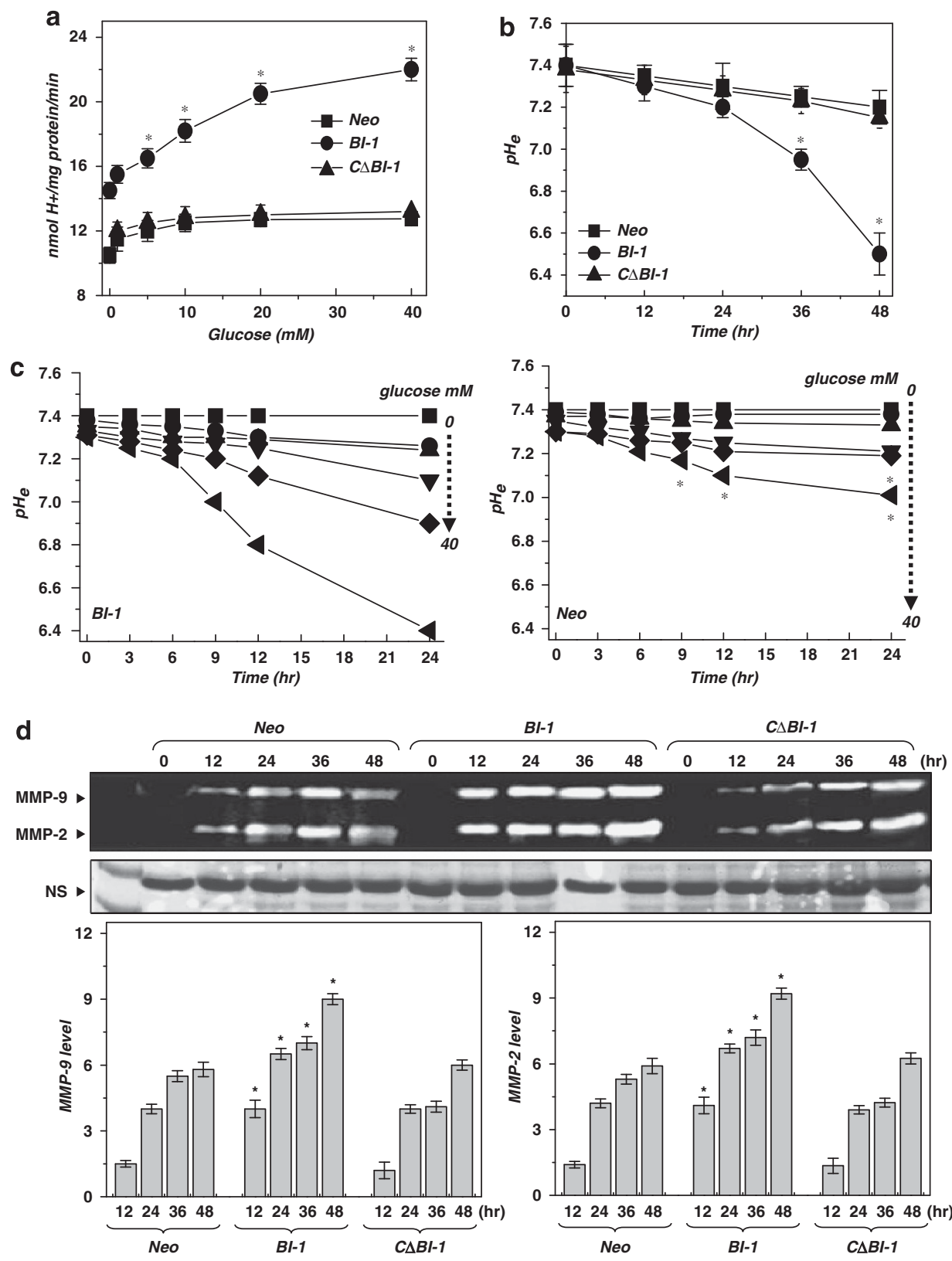

e
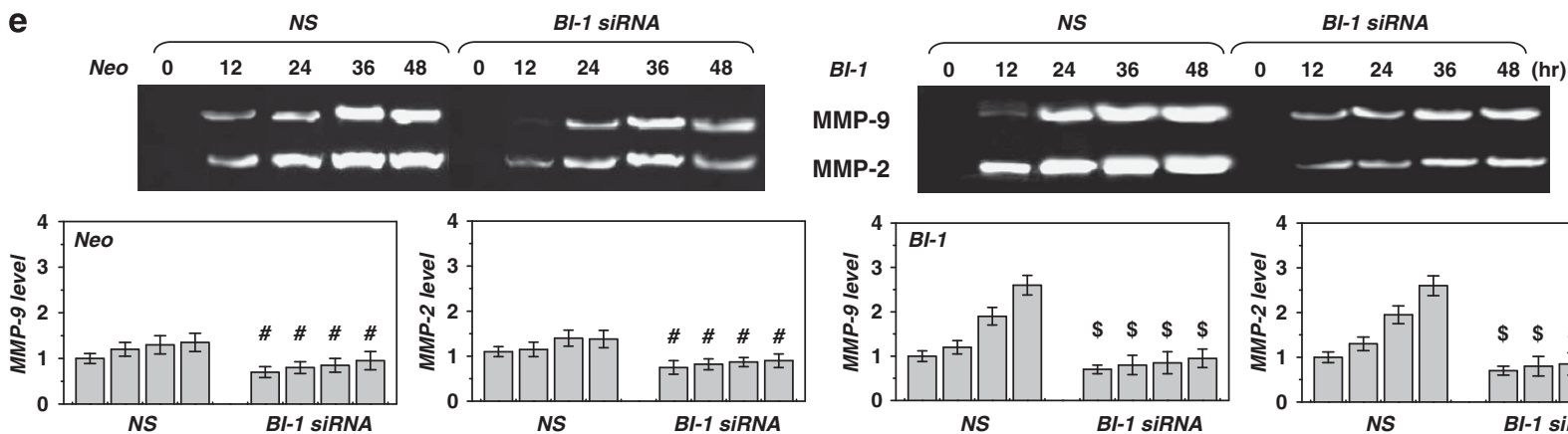

Figure 4.
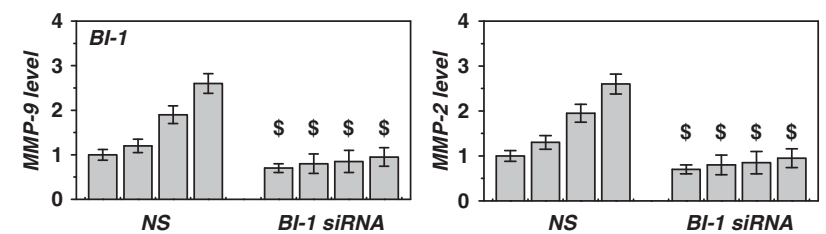